\title{
Pollution des Eaux Lagunaires et Conscience Environnementale des Pecheurs en Cote d'Ivoire
}

\author{
Kodjoh Essoh Steve Gustave, Doctorant
}

Université Félix Houphouët-Boigny, Abidjan, Côte d'Ivoire

Institut d'Ethno-Sociologie, Département de Sociologie. Laboratoire d'Etude

et de Recherche Interdisciplinaires en Sciences Sociales (LERISS)

Doi: 10.19044/esj.2019.v15n6p227 URL:http://dx.doi.org/10.19044/esj.2019.v15n6p227

\section{Résumé}

Les eaux lagunaires du département de Dabou offrent des emplois à de nombreux jeunes déscolarisés et en quête d'emploi par l'exercice de l'activité de la pêche. Toutefois, certains pêcheurs utilisent les produits phytosanitaires comme technique de pêche. Cette étude vise à mettre en exergue la conscience environnementale de ces pêcheurs. A cet effet, l'étude documentaire, l'observation directe et un questionnaire ont été utilisés pour obtenir les données. Pour rendre les résultats objectifs, il a été fait recours à la théorie du choix rationnel. Les résultats ont montré que la pêche aux produits phytosanitaires dans les eaux lagunaires du département de Dabou est pratiquée majoritairement par les autochtones $(88,2 \%)$ et ayant un niveau d'instruction faible tout en sachant lire et écrire $(62,5 \%)$. Les informations obtenues ont permis de constater que la plupart de ces pêcheurs $(78,6 \%)$ ont une expérience professionnelle de plus de 9 ans et ont un faible niveau de conscience environnementale.

Mots-clés: Conscience environnementale, Eaux lagunaires, Pêcheurs, Pollution, Produits phytosanitaires 


\title{
Lagoon Waters Pollution and Environmental Awareness of Fishermen in Ivory Coast
}

\author{
Kodjoh Essoh Steve Gustave, Doctorant
}

Université Félix Houphouët-Boigny, Abidjan, Côte d'Ivoire

Institut d'Ethno-Sociologie, Département de Sociologie. Laboratoire d'Etude et de Recherche Interdisciplinaires en Sciences Sociales (LERISS)

\begin{abstract}
The lagoon waters of the department of Dabou offer jobs to many young people who have dropped out of school and who are looking for work through the exercise of fishing activities. However, some fishermen use plant protection products as a fishing technique. This study aims to highlight the environmental awareness of these fishermen. For this purpose, the desk study, the direct observation and a questionnaire were used to obtain the data. To make the results objective, rational choice theory has been used. The results showed that the fishery for phytosanitary products in the lagoon waters of the Dabou department is practiced mainly by the natives $(88.2 \%)$ and having a low level of education while being able to read and write $(62.5 \%)$. . The information obtained revealed that most of these fishermen $(78.6 \%)$ have a professional experience of more than 9 years and have a low level of environmental awareness.
\end{abstract}

Keywords: Environmental awareness, Lagoon waters, Fishermen, Phytosanitary products, Pollution

\section{Introduction}

Pour Micha et Frank (2004), le poisson représente la principale source de protéine (plus de 70\%) pour les populations africaines. Pour les populations à faible revenu, il est souvent la seule source de protéine accessible. Selon la FAO (1995), la Côte d'Ivoire est l'un des principaux pays consommateurs de protéines de poisson en Afrique de l'Ouest.

La consommation per capita se situe entre 15 et $16 \mathrm{~kg}$ par habitant et par an, soit 50\%, DAP (2014). Dans cette dynamique, la pêche artisanale joue un rôle important avec une contribution de près de $60 \%$ à la production nationale. Cependant, le pays reste fortement dépendant des importations pour la satisfaction de sa demande intérieure en produits halieutiques. La production nationale en poisson est de $50000 \mathrm{t}$ en moyenne alors qu'il y a un besoin de 
plus de 360000 t comblé grâce aux importations, d'une valeur de 289 millions d'euros (DAP, 2014).

Face à la demande très accrue en protéine de poisson, le sous-secteur des pêches est appelé à jouer un rôle primordial. Toutefois, les activités anthropiques interfèrent sur les eaux de pêche en créant des problèmes environnementaux et sanitaires. Les activités domestiques, agricoles, industrielles, hospitalières et les activités de pêche artisanale, sont les plus incriminées. Ainsi, pour Ado et al. (2010), les activités anthropiques provoquent des perturbations de l'équilibre naturel et accroissent la charge organique de l'eau et des sédiments, puis l'encombrement des ressources en eau, avec des problèmes d'eutrophisation, l'asphyxie de l'environnement aquatique ainsi que les problèmes sanitaires des populations.

En effet, pour Keddal et N'Dri (2008), les conséquences de la pollution sur les eaux sont la destruction des ressources vivantes, le déséquilibre des milieux physiques, biologiques et des écosystèmes aquatiques à travers l'eutrophisation, particulièrement dans les zones maritimes ou côtières. Ces situations de pollution des eaux de pêche entraînant la destruction massive des stocks de ressources halieutiques déjà limitées, mettent en mal l'activité de la pêche.

Ainsi, pour accroitre leur rendement certains pêcheurs procèdent à des techniques illicites de pêche telles que l'utilisation des produits phytosanitaires. C'est le cas des eaux lagunaires du département de Dabou. Selon Anoh (2007), dans les milieux lagunaires du littoral ivoirien, le recours aux produits toxiques comme technique de pêche est de plus en plus répandu.

Cette pêche entraîne la surexploitation des ressources halieutiques, la disparition ou la rareté de certaines espèces de poissons qui seraient sensibles aux perturbations du milieu aquatique, tue massivement les œufs de poisson, les alevins et les espèces juvéniles.

A cet effet, des efforts sont faits par les institutions publiques et traditionnelles pour atténuer l'usage des produits phytosanitaires dans la pêche. Mais, force est de constater que certains pêcheurs continuent d'utiliser les produits phytosanitaires de façon récurrente pour pêcher.

De ce fait, une question s'impose à nous: Quel est la conscience environnementale des pêcheurs qui utilisent les produits phytosanitaires pour pêcher?

La réponse à cette question entend se positionner dans une posture épistémologique positiviste et la posture écologique d'un développement conventionnel. 


\section{Methodologie}

Elle consiste à circonscrire les champs de la recherche à travers le site et la population d'étude, l'échantillon, la méthode d'analyse et le positionnement de l'étude.

\subsection{Site et population d'étude}

\subsubsection{Site de l'étude}

Cette étude à été réalisée dans le département de Dabou. Le département de Dabou est situé dans le Sud-est de la Côte d'Ivoire, dans la région des Grands Ponts et à environ $47 \mathrm{Km}$ d'Abidjan la capitale économique. Il est encadré au Sud par le département de Jacqueville, à l'Est ceux de Bingerville et d'Anyama, à l'Ouest par le département de Grand-Lahou et enfin au Nord par le département de Sikensi.

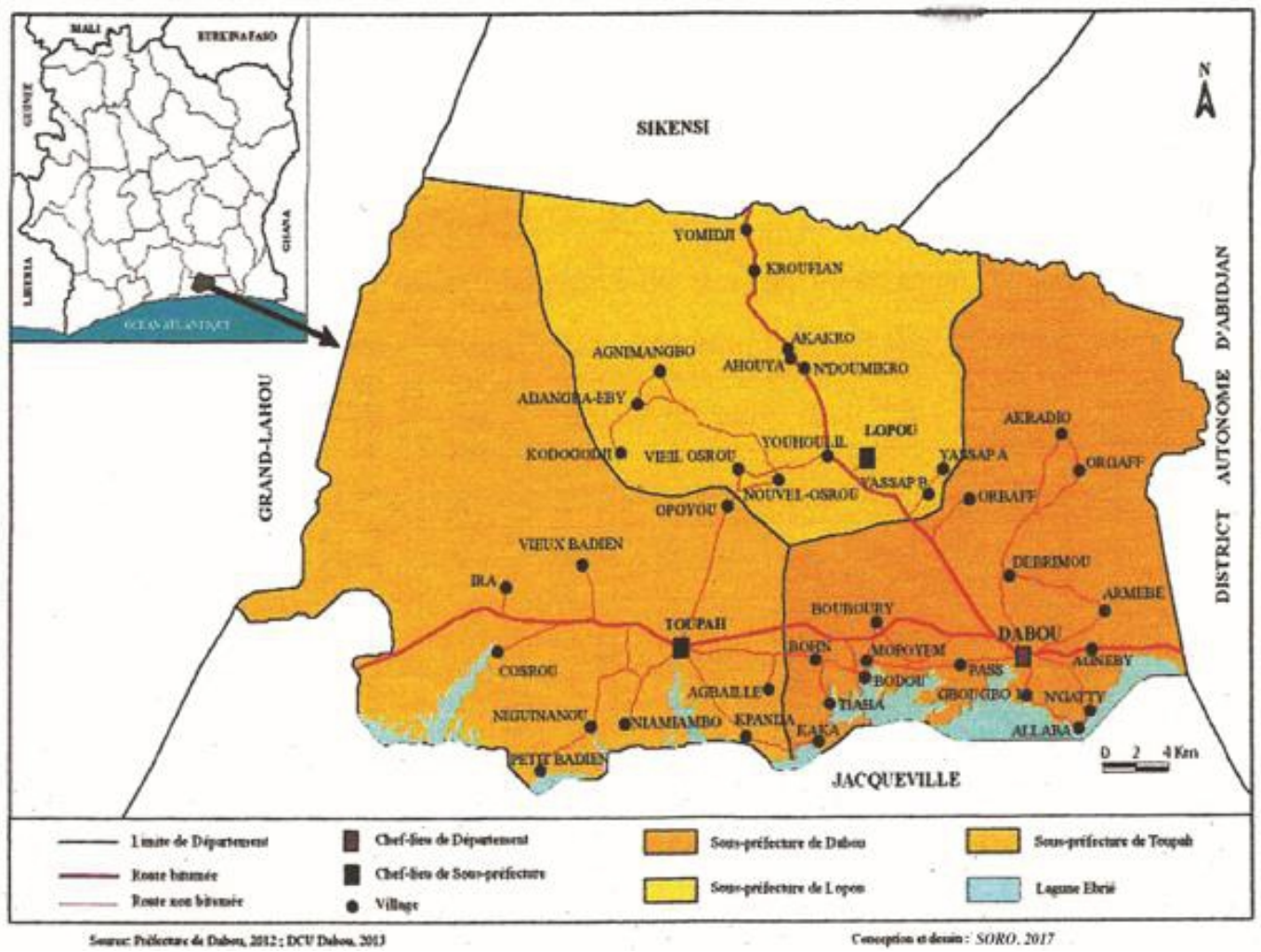

Figure 1 : Situation géographique du département de Dabou

\subsubsection{Population à l'étude}

Cette étude a porté sur les pêcheurs qui utilisent les produits phytosanitaires comme technique de pêche. A cet effet, nous avons fait ressortir leurs caractéristiques socio-démographiques, les différents produits phytosanitaires utilisés et mis en exergue leur conscience environnementale. 


\subsection{L'échantillon}

L'activité de la pêche dans les eaux lagunaires du département de Dabou est une activité informelle qui ne bénéficie d'aucune organisation rationnelle. Aussi, la pêche aux produits phytosanitaires est une activité illicite d'où ces acteurs n'ont fait l'objet d'aucun recensement. En absence de donnée sur le nombre de pêcheurs qui utilisent les produits phytosanitaires comme technique de pêche, nous avons opté pour l'échantillon en boule de neige ou par réseaux. Ce qui donne cent trente-six (136) pêcheurs utilisant les produits phytosanitaires pour pêcher que nous avions interrogé.

\subsection{La méthode d'analyse}

Les données recueillies lors de l'enquête ont fait l'objet d'un dépouillement informatique. A cet effet, nous avons utilisé le logiciel SphinxPlus ${ }^{2} 4.5$ qui a conçu le masque de saisie. Les données ont été transférées sur le logiciel Excel pour calculer les fréquences. Relativement à l'analyse des données, nous avons utilisé l'analyse de contenu quantitatif.

\subsection{Le positionnement de l'étude}

Pour le positionnement de cette étude, le positivisme a été choisi pour la posture épistémologique. Concernant la posture écologique, nous avons opté pour le développement conventionnel. Quant à la posture théorique, la théorie du choix rationnel a été choisie.

\section{Resultats}

\subsection{Caractéristique sociodémographique des pécheurs}

Les caractéristiques sociodémographiques des pêcheurs se perçoivent à travers l'âge, l'origine sociale, le niveau d'instruction, le statut matrimonial et l'expérience professionnelle des pêcheurs.

\subsubsection{Age des pêcheurs}

Le tableau 1 présente l'âge des pêcheurs qui utilisent les produits phytosanitaires.

Tableau 1 : Répartition des pêcheurs selon la tranche d'âge

\begin{tabular}{|l|c|c|}
\hline \multicolumn{1}{|c|}{ Age } & Effectifs & Fréquence en $\%$ \\
\hline [18 ans-25ans [ & 4 & $2,9 \%$ \\
\hline [25 ans-30ans [ & 11 & $8,1 \%$ \\
\hline [30 ans-35ans [ & 15 & $11,0 \%$ \\
\hline [35 ans-40ans [ & 46 & $33,8 \%$ \\
\hline [40 ans-45ans [ & 58 & $42,6 \%$ \\
\hline [45 ans-50ans] & 2 & $1,5 \%$ \\
\hline TOTAL & 136 & $100 \%$ \\
\hline
\end{tabular}

Source : Enquête de terrain, août 2016-avril 2017 
Le tableau indique que sur les 136 pêcheurs interrogés, 121 pêcheurs (soit $89 \%$ des enquêtés) ont un âge compris entre 30 et 50 ans. Ceux-ci sont suivis de ceux dont l'âge varie entre 18 et 30 ans et qui représentent $11 \%$ de la population.

\subsubsection{L'origine sociale des pêcheurs}

Par "origine sociale", nous entendons ici, la nationalité et le pays d'origine des pêcheurs. Mais, nous utiliserons surtout les notions d'autochtone, allogène et allochtone pour identifier nos enquêtés étant donné que cette recherche a été réalisée en zone rurale.

Tableau 2: Répartition des enquêtés selon l'origine sociale

\begin{tabular}{|l||c||c|}
\hline \multicolumn{1}{|c||}{ Communauté } & Effectifs & Fréquence en $\%$ \\
\hline Autochtone & 120 & $88,2 \%$ \\
\hline Allogène & 13 & $9,6 \%$ \\
\hline Allochtone & 3 & $2,2 \%$ \\
\hline TOTAL & 136 & $100 \%$ \\
\hline
\end{tabular}

Source : Enquête de terrain, août 2016-avril 2017

Il apparait à la lecture de ce tableau que sur les 136 pêcheurs interrogés, 120 pêcheurs (soit 88,2\% des enquêtés) sont des autochtones Adjoukrou. On a dénombré 13 pêcheurs allogènes (soit 9,6\% des enquêtés). Les pêcheurs allochtones quant à eux, sont 3 et représentent 2,2\% des enquêtés.

Cette forte proportion des autochtones Adjoukrou (88,2\%) s'explique par le fait que, les Adjoukrou ont toujours maintenu la tradition de la pêche malgré l'influence de l'urbanisation et des cultures de rente telles que; le palmier à huile, l'hévéa et le binôme café-cacao qui sont des sources de revenus très considérables. D'autre part, les autochtones Adjoukrou ont restreint l'accès à leur plan d'eau lagunaire aux étrangers par le constat de 'tradition pêcheur".

\subsubsection{Le niveau d'instruction des pêcheurs}

Tableau 3: Répartition des enquêtés selon le niveau d'instruction

\begin{tabular}{|l||c||c|}
\hline \multicolumn{1}{|c||}{ Niveau d'étude } & Effectifs & Fréquence en\% \\
\hline Analphabète & 2 & $1,5 \%$ \\
\hline Primaire (y compris le CEPE) & 83 & $61 \%$ \\
\hline Secondaire (1er cycle compris le BEPC) & 40 & $29,4 \%$ \\
\hline Secondaire (2ème cycle compris le BAC) & 10 & $7,4 \%$ \\
\hline Niveau supérieur 1er cycle (BAC+2) & 1 & $0,7 \%$ \\
\hline TOTAL & 136 & $100 \%$ \\
\hline \hline
\end{tabular}

Source : Enquête de terrain, août 2016- avril 2017 
L'enquête réalisée auprès des pêcheurs a permis de faire ressortir leur niveau d'instruction. Le tableau indique que 2 pêcheurs (soit 1,5\% des enquêtés) sont des analphabètes et 83 pêcheurs (soit $61 \%$ des enquêtés) ont un niveau primaire, soit $62,5 \%$ ont un niveau d'instruction relativement faible, mais tout en sachant lire et écrire. On note aussi que 50 pêcheurs (soit 36,8\% des enquêtés) ont un niveau secondaire ( $1^{\text {er }}$ et 2ème cycle), et seulement 1 pêcheur (soit $0,7 \%$ des enquêtés) a un niveau supérieur.

La prédominance des pêcheurs à faible niveau d'instruction peut s'expliquer par le fait que ce secteur d'activité ne requiert pas ou n'exige un niveau élevé ou très élevé d'instruction. Tout le monde peut s'adonner à l'activité de la pêche à condition de maîtriser quelque technique de pêche. Ce qui expliquerait pourquoi ce secteur attire beaucoup plus de personnes non diplômées.

\subsubsection{Le statut matrimonial des pêcheurs}

Tableau 4: Répartition des enquêtés selon le statut matrimonial

\begin{tabular}{|l|c||c|}
\hline \hline Statut matrimonial & Effectifs & Fréquence en $\%$ \\
\hline Célibataire & 19 & $14,0 \%$ \\
\hline Marié & 106 & $77,9 \%$ \\
\hline Divorcé/Séparé & 9 & $6,6 \%$ \\
\hline Veuf & 2 & $1,5 \%$ \\
\hline TOTAL & 136 & $100 \%$ \\
\hline
\end{tabular}

Source : Enquête de terrain, août 2016- avril 2017

Selon notre tableau 106 pêcheurs (soit 77,9\% des enquêtés) sont des mariés, 19 pêcheurs (soit $14 \%$ des enquêtés) sont des célibataires et les divorcés représentent $6,6 \%$ de nos enquêtés.

\subsubsection{Expérience professionnelle des pêcheurs}

Tableau 5: Répartition des enquêtés selon l'expérience professionnelle

\begin{tabular}{|c||c||c|}
\hline \hline Expérience professionnelle & Effectifs & Fréquence en\% \\
\hline Moins de 3ans & 0 & $0,0 \%$ \\
\hline [3ans-5ans] & 2 & $1,5 \%$ \\
\hline [6ans-8ans] & 27 & $19,9 \%$ \\
\hline [9ans-11ans] & 27 & $19,9 \%$ \\
\hline [12ans-14ans] & 21 & $15,4 \%$ \\
\hline [15ans-17ans] & 32 & $23,5 \%$ \\
\hline [18ans-20ans] & 14 & $10,3 \%$ \\
\hline [21ans et Plus [ & 13 & $9,6 \%$ \\
\hline
\end{tabular}




\begin{tabular}{|c|c|c|}
\hline TOTAL & 136 & $100 \%$ \\
\hline
\end{tabular}

Source : Enquête de terrain, août 2016- avril 2017

La classe modale de cette série statistique est [15ans-17ans]. Ce qui veut dire que, la plupart des pêcheurs (32 pêcheurs soit 23,5\%) qui utilisent les produits ont une expérience professionnelle allant de 15 à 17 ans. Ces données statistiques montrent que $78,6 \%$ des pêcheurs interrogés ont une expérience professionnelle de plus de 9 ans, contre 21,4\% qui ont moins de 9 ans d'expériences professionnelles.

Ainsi, il en résulte que, la pêche aux produits phytosanitaires est le fait de pêcheurs expérimentés.

\subsection{La conscience environnementale des pêcheurs qui utilisent les produits phytosanitaires}

\subsubsection{Les produits phytosanitaires utilisés par les pêcheurs}

Le tableau ci-dessous liste les différents produits phytosanitaires utilisés par les pêcheurs dans les eaux lagunaires du département de Dabou.

Tableau 6: Répartition des enquêtés selon les produits phytosanitaires utilisés

\begin{tabular}{|c|c|c|}
\hline Produits phytosanitairesutilisés & Effectifs & Fréquence en $\%$ \\
\hline Thiodan c 50 & 47 & $34,6 \%$ \\
\hline Callifan e 50 & 63 & $46,3 \%$ \\
\hline Décis 480 ec & 116 & $85,3 \%$ \\
\hline Stater & 35 & $25,7 \%$ \\
\hline Marshal 480 ec & 130 & $95,6 \%$ \\
\hline Faser & 53 & $39,0 \%$ \\
\hline Endocotton & 34 & $25,0 \%$ \\
\hline Tango tango & 56 & $41,2 \%$ \\
\hline Roukis & 53 & $39,0 \%$ \\
\hline TOTAL & 136 & \\
\hline
\end{tabular}

Source : Enquête de terrain, août 2016- avril 2017

Comme l'indique notre tableau, plusieurs produits phytosanitaires employés en agriculture sont utilisés par certains pêcheurs dans les eaux lagunaires du département de Dabou. Les produits phytosanitaires les plus utilisés sont le Marshall 480 ec avec 95,6\% d'utilisation, le Décis 480 ec avec $85,3 \%$ d'utilisation et le Callifan e 50 avec $46,3 \%$ d'utilisation.

\subsubsection{Nombre d'année d'utilisation des produits phytosanitaires}

Cette variable nous permet de savoir si le temps à un impact sur la conscience environnementale des pêcheurs, compte tenu des conséquences de 
la pêche aux produits phytosanitaires sur la faune aquatique et sur les êtres humains.

Tableau 7: Répartition des enquêtés selon le nombre d'année d'utilisation des produits phytosanitaires

\begin{tabular}{|l|c||c|}
\hline $\begin{array}{c}\text { Nombre d'année d'utilisation des } \\
\text { produits phytosanitaires }\end{array}$ & Effectifs & $\begin{array}{c}\text { Fréquence } \\
\text { en\% }\end{array}$ \\
\hline [1an-2ans] & 18 & $13,2 \%$ \\
\hline [3ans-4ans] & 40 & $29,4 \%$ \\
\hline [5ans-6ans] & 43 & $31,6 \%$ \\
\hline [7ans-8ans] & 28 & $20,6 \%$ \\
\hline [9ans-10ans] & 7 & $5,1 \%$ \\
\hline TOTAL & 136 & $100 \%$ \\
\hline \hline
\end{tabular}

Source : Enquête de terrain, août 2016-avril 2017

La classe modale de cette série statistique est l'intervalle [5ans-6ans]. Ce qui veut dire que cela fait en moyenne cinq à six ans que ces pêcheurs utilisent les produits phytosanitaires pour pêcher.

Le tableau indique que sur les 136 pêcheurs interrogés, 58 pêcheurs $(18+40)$, soit $42,6 \%$ des enquêtés pratiquent la pêche aux produits phytosanitaires il y a moins de 5 ans et 78 pêcheurs $(43+28+7)$, soit $57,4 \%$ des enquêtés, pêchent avec les produits phytosanitaires il y a de cela 5 à 10 ans.

\subsubsection{Connaissance de la destruction massive des oufs de poissons, des alevins et des juvéniles}

Tableau 8: Connaissance de la destruction massive des œufs de poissons, des alevins et des juvéniles

\begin{tabular}{|l||c|c|}
\hline \multicolumn{1}{|c|}{ Modalités } & Effectifs & Fréquence en $\%$ \\
\hline Oui & 133 & $97,8 \%$ \\
\hline Non & 0 & $0,0 \%$ \\
\hline Sans réponse & 3 & $2,2 \%$ \\
\hline TOTAL & 136 & $100 \%$ \\
\hline
\end{tabular}

Source : Enquête de terrain, août 2016- avril 2017

Selon notre tableau, sur les 136 pêcheurs enquêtés, 133 pêcheurs (soit 97,8\% des enquêtés) affirment savoir que la pêche aux produits phytosanitaires tue massivement les espèces juvéniles, les alevins et les œufs de poissons. On a seulement 3 pêcheurs (soit 2,2\% des enquêtés) qui demeurent sans réponse sur cette question. 
Il ressort de cette analyse que, la plupart des pêcheurs $(97,8 \%)$ savent bien que la pêche aux produits phytosanitaires tue massivement les ressources halieutiques.

\subsubsection{Connaissance de la quantité de poissons dissipés}

La quantité de poissons dissipés représente ici tout le reste de poissons que les pêcheurs n'arrivent pas à capturer et qui meurt dans l'eau quelles que heures après la pêche. Cette quantité de poissons serait plus importante que celle que les pêcheurs arrivent à capturer. Nous demandons leur avis sur cette question.

Tableau 9: Répartition des enquêtés selon la connaissance de la quantité de poissons dissipés

\begin{tabular}{|l||c||c|}
\hline \multicolumn{1}{|c|}{ Modalités } & Effectifs & Fréquence en $\%$ \\
\hline Vrai & 109 & $80,1 \%$ \\
\hline Faux & 19 & $14,0 \%$ \\
\hline Ne sais pas & 8 & $5,9 \%$ \\
\hline TOTAL & 136 & $100 \%$ \\
\hline \hline
\end{tabular}

Source : Enquête de terrain, août 2016- avril 2017

Le tableau indique que sur les 136 pêcheurs interrogés, 109 pêcheurs (soit $80,1 \%$ des enquêtés) affirment qu'il est vrai que la quantité de poissons qu'ils n'arrivent pas à capturer et qui meurt dans l'eau est largement élevée que celle qu'ils arrivent à pêcher. Par contre, 19 pêcheurs (soit $14,0 \%$ des enquêtés) soutiennent le contraire. Les pêcheurs déclarant ne pas savoir si la quantité de poissons qu'ils n'arrivent pas à capturer et qui meurt dans l'eau est plus élevée que celle qu'ils arrivent à pêcher représentent 5,9\% des enquêtés (soit 8 pêcheurs).

\subsubsection{Connaissance des zones de frayère et de nursery des poissons}

Les zones de frayère et de nursery sont respectivement des lieux aquatiques où les poissons assurent leur reproduction et où les juvéniles se concentrent pour se nourrir. L'utilisation des produits phytosanitaires dans ces lieux va causer la disparition des espèces les plus vulnérables et aussi limiter le renouvellement des ressources halieutiques. Cette variable permet de mettre en évidence la lucidité des pêcheurs sur l'environnement aquatique.

Tableau 10: Répartition des enquêtés selon la connaissance des zones de frayère et de nursery des poissons

\begin{tabular}{|l||c||c|}
\hline \multicolumn{1}{|c|}{ Modalités } & Effectifs & Fréquence en $\%$ \\
\hline Oui & 136 & $100 \%$ \\
\hline Non & 0 & $0,0 \%$ \\
\hline Sans réponse & 0 & $0,0 \%$ \\
\hline
\end{tabular}




\begin{tabular}{|l||c|c|}
\hline TOTAL & 136 & $100 \%$ \\
\hline
\end{tabular}

Source : Enquête de terrain, août 2016- avril 2017

De ce tableau, il ressort que tous les enquêtés (100\%) savent que les roseaux et les lieux rocheux où ils pêchent avec les produits phytosanitaires sont des zones de frayère et de nursery des poissons.

Il en résulte que, tous les pêcheurs ne tiennent absolument pas compte des graves conséquences de l'utilisation des produits phytosanitaires dans les zones de frayère et de nursery des poissons.

\subsubsection{Connaissance de la durée de pollution des zones polluées}

Les espaces de pêche où les pêcheurs utilisent les produits phytosanitaires pour pêcher seraient contaminés pour une durée d'au moins deux mois. A cet effet, nous cherchons à savoir si les pêcheurs ont la connaissance de cette duré de pollution de ces espaces de pêche.

Tableau 11: Répartition des enquêtés selon la connaissance de la durée de pollution des espaces de pêche pollués

\begin{tabular}{|l||c||c|}
\hline \multicolumn{1}{|c|}{ Modalités } & Effectifs & Fréquence en $\%$ \\
\hline Oui & 135 & $99,3 \%$ \\
\hline Non & 1 & $0,7 \%$ \\
\hline Sans réponse & 0 & $0,0 \%$ \\
\hline TOTAL & 136 & $100 \%$ \\
\hline
\end{tabular}

Source : Enquête de terrain, août 2016- avril 2017

Le tableau indique que, sur les 136 pêcheurs interrogés, 135 pêcheurs (soit 99,3\% des enquêtés) affirment qu'ils savent que les endroits où ils utilisent les produits phytosanitaires pour pêcher sont pollués pour au moins deux (02) mois, contre seulement 1 pêcheurs (soit $0,7 \%$ des enquêtés) qui soutient ne pas savoir.

\subsubsection{Connaissance d'espèces de poissons devenues rares ou disparues en rapport avec l'utilisation des produits phytosanitaires}

Notre enquête a révélé que plusieurs espèces de poissons sont devenues rares ou ont disparu dans les eaux lagunaires du fait de l'utilisation récurrente des produits phytosanitaires dans la pêche.

Tableau 12: Répartition des enquêtés selon que la pêche aux produits phytosanitaires entraîne la rareté ou la disparition des espèces de poissons

\begin{tabular}{|l|c||c|}
\hline \multicolumn{1}{|c|}{ Modalités } & Effectifs & Fréquence en \% \\
\hline Oui & 67 & $49,3 \%$ \\
\hline Non & 61 & $44,9 \%$ \\
\hline Ne sais pas & 8 & $5,9 \%$ \\
\hline
\end{tabular}




\begin{tabular}{|l|c|c|}
\hline TOTAL & 136 & $100 \%$ \\
\hline
\end{tabular}

Source : Enquête de terrain, août 2016- avril 2017

Les données du tableau, nous révèlent que sur les 136 pêcheurs enquêtés, 67 pêcheurs (soit 49,3\% des enquêtés) soutiennent que la rareté ou la disparition des espèces de poissons est due à l'utilisation récurrente des produits phytosanitaires dans la pêche. Contre 61 pêcheurs, soit $44,9 \%$ des enquêtés qui affirment le contraire. Les pêcheurs qui ne savent pas si la pêche aux produits phytosanitaires est la cause de la rareté ou la disparition des espèces de poissons sont au nombre de 8 et représentent $5,9 \%$ des enquêtés.

Photo 1: Exemple de poissons en voie de disparition dans les eaux lagunaires du

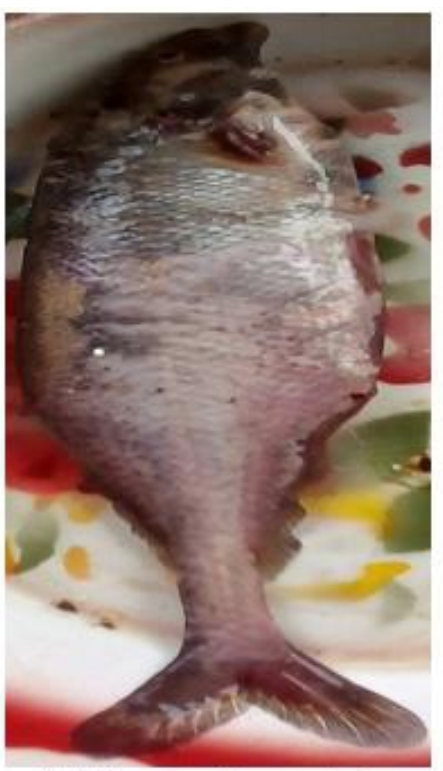

A: Marcusenius ussheri

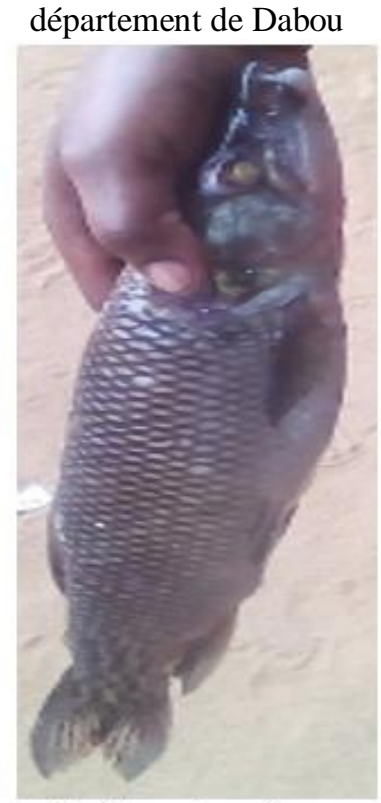

B: Hepsetus odoe

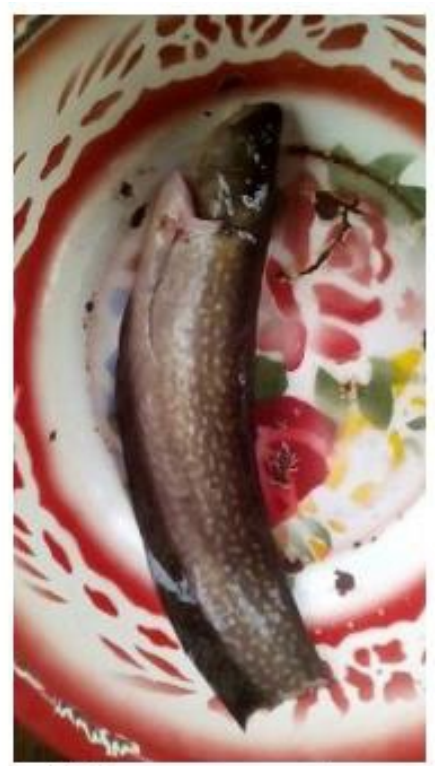

C: Papyrocranus afer

Source : Données de l'enquête de terrain, août 2016- avril 2017

\subsubsection{Connaissance des effets de la consommation des ressources halieutiques pêchées à l'aide de produits phytosanitaires}

Cette rubrique nous situe sur la connaissance des pêcheurs aux effets de la consommation des ressources halieutiques qu'ils pêchent avec les produits phytosanitaires sur la santé des populations.

Tableau 13: Répartition des enquêtés selon la connaissance des effets de la consommation des ressources halieutiques pêchées avec des produits phytosanitaires

\begin{tabular}{|l||c|c|}
\hline \multicolumn{1}{|c|}{ Modalités } & Effectifs & Fréquence en $\%$ \\
\hline Oui & 125 & $91,9 \%$ \\
\hline Non & 11 & $8,1 \%$ \\
\hline Sans réponse & 0 & $0,0 \%$ \\
\hline
\end{tabular}




\begin{tabular}{|l||c|c|}
\hline TOTAL & 136 & $100 \%$ \\
\hline
\end{tabular}

Source : Enquête de terrain, août 2016- avril 2017

Le tableau montre que sur les 136 pêcheurs interrogés, 125 pêcheurs, soit $91,9 \%$ des enquêtés répondent par l'affirmation savoir que, la consommation des ressources halieutiques pêchées avec les produits phytosanitaires a des répercutions sur la santé, contre seulement 11 pêcheurs, soit $8,1 \%$ des enquêtés qui soutiennent ne pas savoir.

\subsubsection{Perception de la pêche aux produits phytosanitaires}

Tableau 14: Répartition des pêcheurs selon la perception de l'utilité des produits phytosanitaires dans la pêche

\begin{tabular}{|l|c||c|}
\hline \multicolumn{1}{|c|}{ Réponses } & Effectifs & Fréquence en\% \\
\hline Permet de capturer facilement les gros tilapias & 58 & $42,6 \%$ \\
\hline La pêche au produit phytosanitaire est rapide & 36 & $26,5 \%$ \\
\hline La pêche au produit phytosanitaire n'est pas pénible & 33 & $24,3 \%$ \\
\hline Permet de capturer plus de poissons (tilapias) & 136 & $100 \%$ \\
\hline TOTAL & 136 & \\
\hline \hline
\end{tabular}

Source : Enquête de terrain, août 2016- avril 2017

A partir de l'inventaire statistique établi dans ce tableau, nous remarquons bien que tous les pêcheurs (100\%) s'accordent pour dire que, les produits phytosanitaires permettent de capturer plus de tilapias. Aussi, 58 pêcheurs (soit 42,6\% des enquêtés) affirment que la pêche aux produits phytosanitaires permet de capturer facilement les gros tilapias. 36 pêcheurs (soit $26,5 \%$ des enquêtés) et 33 pêcheurs (soit $24,3 \%$ des enquêtés) disent respectivement que la pêche aux produits phytosanitaires est rapide et n'est pas pénible.

Ces données traduisent le fait que les techniques de pêche traditionnelles pratiquées par les pêcheurs enquêtés seraient pénibles, voir très fatigantes, et ne permettraient pas de capturer suffisamment les tilapias. Ainsi, les produits phytosanitaires seraient plus efficaces pour augmenter leur rendement. Ce qui expliquerait leur utilisation récurrente dans la pêche.

\section{Discussion}

Au vue des résultats obtenus par l'analyse de nos différentes données, il est ressorti une présence massive des adultes (90\%) dans l'utilisation des produits phytosanitaires dans la pêche et ayant une expérience professionnelle de plus de 9 ans (78,6\%). Contrairement à nous, Vanga (2013), qui a écrit sur "Acteurs locaux et pêche lagunaire aux produits toxiques dans la souspréfecture de Grand-Bassam (Côte d'Ivoire) », soutient plutôt une présence 
massive des jeunes dans la pêche $(52 \%)$ et inexpérimentés (52\% ayant moins de 10 années de pratiques dans la pêche). Et ce fait trouve son explication principale dans le chômage en ville. Ces jeunes et adultes viennent au village pour s'insérer dans un secteur d'activité générateur de revenus. A partir de ce moment, la pêche s'est présentée comme une alternative pour les populations riveraines au chômage et sans sources de revenus.

Relativement à l'origine sociale des pêcheurs qui utilisent les produits phytosanitaires pour pêcher, il ressort que ces pêcheurs sont en majorité des autochtones Adjoukrou (88,2\%). Sur ce point, notre résultat va dans le même sens que celui de Vanga (2013). Il soutient que les pêcheurs sont en majorité des autochtones (77\%). Même si les allochtones et les allogènes sont présents dans l'activité, la pêche est avant tout une activité du «village» qui bénéficie d'une longue tradition de pêche.

D'après l'analyse du niveau d'instruction de ces pêcheurs, nous allons dans le même sens que Vanga (2013), pour soutenir que, ce sont des pêcheurs qui ont un faible niveau d'instruction mais tout en sachant lire et écrire (62,5\%). Contrairement à nous deux, Boguhé et al, (2011) qui ont travaillés sur la pêche crevettière du fleuve Bandama (Côte d'Ivoire), soutiennent que les pêcheurs sont généralement analphabètes.

Le fait que la plupart des pêcheurs sachent lire et écrire, devrait constituer un atout majeur pour une gestion durable de la pêche lagunaire. Mais, nos données montrent que ces pêcheurs utilisent de façon récurrente les produits phytosanitaires pour pêcher même dans les zones de frayère et de nurserie des poissons.

Pour ce qui concerne les produits phytosanitaires utilisés, les résultats ont montré que plusieurs produits phytosanitaires employés en agriculture sont utilisés par les pêcheurs. Les produits phytosanitaires les plus utilisés sont le Marshall 480 ec avec 95,6\% d'utilisation, le Décis 480 ec avec 85,3\% d'utilisation et le Callifan e 50 avec 46,3\% d'utilisation. Vanga (2013) qui va dans le même sens que nous, soutient que les produits toxiques phytosanitaires employés en agriculture constituent l'essentiel des substances utilisées dans la pêche lagunaire au niveau de la Sous-préfecture de Grand-Bassam. Il fait la typologie de ces produits qui sont entre autres : des pesticides, des insecticides, des herbicides et autres. A ce niveau, pour lui, ce sont les pesticides qui sont utilisés de manière significative $(65 \%)$.

Cette recherche a montré aussi que, presque tous les pêcheurs $(97,8 \%)$, savent que les produits phytosanitaires occasionnent la destruction massive des œufs de poissons, des alevins et des espèces juvéniles. De même, 99,3\% déclarent être savoir que les endroits où ils utilisent les produits phytosanitaires pour pêcher sont pollués pour au moins deux (02) mois. Dans cette même perspective, Vanga (2013), soutient que l'utilisation des produits toxiques pour la pêche en lagune pollue l'eau sur une distance importante. Des 
milliers de poissons, de crustacés et de reptiles se retrouvent pris au piège de cette pratique. Pour lui, cela contribuera à appauvrir davantage le plan d'eau lagunaire, qui s'est vidé progressivement de ses ressources. Egalement, presque tous les pêcheurs $(94,1 \%)$ savent que plusieurs espèces de poissons sont devenues rares ou ont disparu. Pour 49, 3\% d'entre eux, cela est dû à l'utilisation des produits phytosanitaires. Dans cette perspective, Karaki et $a l$. (1997), relèvent que l'accumulation des pesticides dans les œufs entraîne une mortalité élevée des alevins et des troubles métaboliques graves qui peuvent conduire à l' extermination de certaines espèces. Quant à Etien et Dadié (1992), ils soutiennent que des pesticides comme le Lindane (Gammaline) et le thiodan (Endosulfan) agissent sur l'œil, les branchies, la bouche, les nageoires, les organes internes et la coloration de la peau des poissons appartenant aux espèces Chrysichtys nigrodigitatus et tilapia aurea.

Par ailleurs, notre étude a relevé plusieurs cas d'intoxications alimentaires dus à la consommation de ces poissons et crustacés pêchés à l'aide de produits phytosanitaires. Et plus de la majorité des pêcheurs $(89 \%)$ affirme être conscient des cas d'intoxications alimentaires de plusieurs personnes ayant consommé les poissons et les crustacés pêchés avec les aux produits phytosanitaires. Pour Vanga (2013), la consommation de poissons empoisonnés, appelés localement 'Zolio', a des répercussions sur la santé des consommateurs. Il affirme dans son étude que, les enquêtés, pour la plupart, estiment que cette consommation provoque des diarrhées et des vomissements chez les concernés. Quant à Olishifski (1979), cité par Anoh (2007), les hydrocarbonés chlorés ont des effets sur le foie, les reins et le système nerveux de l'homme. Par ailleurs, ils sont cancérigènes et peuvent plonger l'homme dans un coma et le conduire à la mort.

\section{Conclusion}

Cette étude a été menée dans le département de Dabou et a porté sur le sujet : «Pollution des eaux lagunaires et conscience environnementale des pêcheurs en côte d'ivoire. » La préoccupation dans ce travail a été de mettre en exergue la conscience environnementale des pêcheurs qui utilisent les produits phytosanitaires dans les eaux lagunaires comme technique de pêche.

Pour rendre les résultats de cette étude plus objectifs, l'observation directe, le questionnaire et l'échantillonnage ont été utilisés pour collecter les données. Quant à l'analyse des données nous avons utilisé l'analyse de contenu quantitatif.

L'étude a révélé que: la pêche aux produits phytosanitaires est principalement pratiquée par les pêcheurs autochtones. Ces pêcheurs sont en majorité des adultes et ayant une expérience professionnelle de plus de 9 ans. La grande majorité de ces pêcheurs a un niveau faible d'instruction mais tout en sachant lire et écrire. En ce qui concerne la conscience environnementale 
des pêcheurs, les résultats ont montré que presque tous les pêcheurs savent que la pêche aux produits phytosanitaires tue massivement les œufs de poissons, les alevins et les espèces juvéniles. De même, la plupart des pêcheurs sait que plusieurs espèces de poissons sont devenues rares ou ont disparu. Egalement, les résultats ont révélé que plus de la majorité des enquêtés affirme qu'il est vrai que la quantité de poissons qu'ils n'arrivent pas à capturer et qui meurt dans l'eau est largement élevée que celle qu'ils arrivent à pêcher. Tous les enquêtés savent que les roseaux et les lieux rocheux où ils pêchent avec les produits phytosanitaires sont des zones de frayère et de nursery des poissons et affirment savoir que ces endroits sont pollués pour au moins deux (02) mois. $\mathrm{Au}$ vue de ces résultats présentés, il ressort que les pêcheurs ont un faible niveau de conscience environnementale. Ce qui expliquerait l'utilisation récurrente des produits phytosanitaires dans la pêche.

\section{References:}

1. ADO, G. et al. (2000): Etude statistique du rôle du phosphore et de l'azote NTK dans le mécanisme de l'eutrophisation des lacs de la ville de Yamoussoukro en Côte d'Ivoire. J. Soc. Ouest - Afr. Chim., 10 : pages $155-171$.

2. ANOH K. P., (2007) : Impact environnemental et socioéconomique de la pêche par empoisonnement en milieu littoral ivoirien. Revue de Géographie Tropicale et d'Environnement, $\mathrm{n}^{\circ} 2$ : page 2 - 13.

3. BOGUHE G. D. et al, (2011): Premières données sur la pêche crevettière du fleuve Bandama (Côte d'Ivoire) : Acteurs et Engins de pêche. Sciences \& Nature, Vol. 8, N¹ : pages 107 - 118.

4. ETIEN N. et DADIE P., (1992) : Définition de critères anatomopathologiques de certains pesticides sur les poissons. Ministère de l'Agriculture et des Ressources Animales, RAP. Direction des Pêches 15 pages.

5. FAO, (2016) : La situation mondiale des pêches et de l'aquaculture. Contribuer à la sécurité alimentaire et à la nutrition de tous. Rome. 224 pages.

6. FAO, (1995) : Code de conduite pour une pêche responsable, FAO, Rome, 46 pages.

7. Institut National de la Statistique (INS), (2014): Annuaire des Statistiques Démographiques et Sociales, Edition N7 : 2007-2010.

8. KARAKI H.et al, (1997): Evaluation des teneurs en pesticides organochlorés dans la région de Buyo à travers les matrices poisson, sédiment et sol : Etude préliminaire, 3èmes journées annuelles de la soachim, Lomé, page 46-51. 
9. KEDDAL, H. et N'DRI, J.Y. (2008) : Impacts de l'intensification agricole sur la qualité des eaux de surface et des eaux souterraines. Revue HTE N ${ }^{\circ} 138$, Sept-Déce. 2007.

10. MICHA J.C. et Franck V., (2004) : Etude prospective pour la relance $d u$ secteur pêche et aquaculture en côte d'Ivoire. Ministère de la Production Animale et des Ressources Halieutiques, Abidjan, 60 p.

11. OLINSHIFSKI B. J., (1979): Fundamentals of industrial hygiene, 2nd edition, National Safety Council, Chicago, Illinois, pages 1023-1117.

12. VANGA. F. A., (2013): Acteurs locaux et pêche lagunaire aux produits toxiques dans la sous-préfecture de Grand-Bassam (Côte d'Ivoire). Agronomie Africaine, 25 (3) : pages 299-308. 